\title{
Effective eddy viscosities in implicit large eddy simulations of turbulent flows
}

\author{
J. Andrzej Domaradzki \\ Department of Aerospace and Mechanical Engineering, \\ University of Southern California, \\ Los Angeles, CA 90089-1191, U.S.A., \\ tel. 213-740-5357, FAX 213-740-777\%4
}

Solving Navier-Stokes equations using monotone algorithms often produces results that are consistent with the dynamics of high Reynolds number turbulence despite formally insufficient numerical resolution to capture all physically relevant scales of motion. It is frequently argued that the truncation error of a numerical scheme provides a mechanism for the energy dissipation and serves as an implicit subgrid scale model. Such an approach to turbulence modeling, known loosely as the Monotonically Integrated LES (MILES), was originally proposed by Boris et al. (1992) and reviewed recently by Grinstein and Fureby (2002). The primary effect of a numerical discretization in MILES, which is similar to the effects of explicit SGS models, is the dissipation of turbulent kinetic energy. Despite its importance, very little is known about details of numerical dissipation in MILES methods and, more importantly, its relation (or lack of it) to the actual dissipative effects of turbulence. We describe a method for computing effective numerical eddy viscosity, useful for assessing numerical dissipation of such approaches. The method is evaluated on an example of a specific nonoscillatory finite volume scheme MPDATA developed for simulations of geophysical flows. A series of numerical simulations of homogeneous, isotropic turbulence are performed and the numerical eddy viscosities are determined. The detailed quantitative comparisons are made between the numerical eddy viscosities and the theoretical eddy viscosity as well as intrinsic eddy viscosities computed exactly from the velocity fields by introducing artificial wave number cutoffs. A typical example of such a comparison is shown in the enclosed figure.

[1] J. P. Boris, F. F. Grinstein, E. S. Oran, and R. L. Kolbe. New insights into large eddy simulation. Fluid Dynamics Research, 10:199-228, 1992.

[2] F.F. Grinstein and C. Fureby. Recent progress on MILES for high Reynolds number flows. J. Fluids Eng., 124:848-861, 2002.

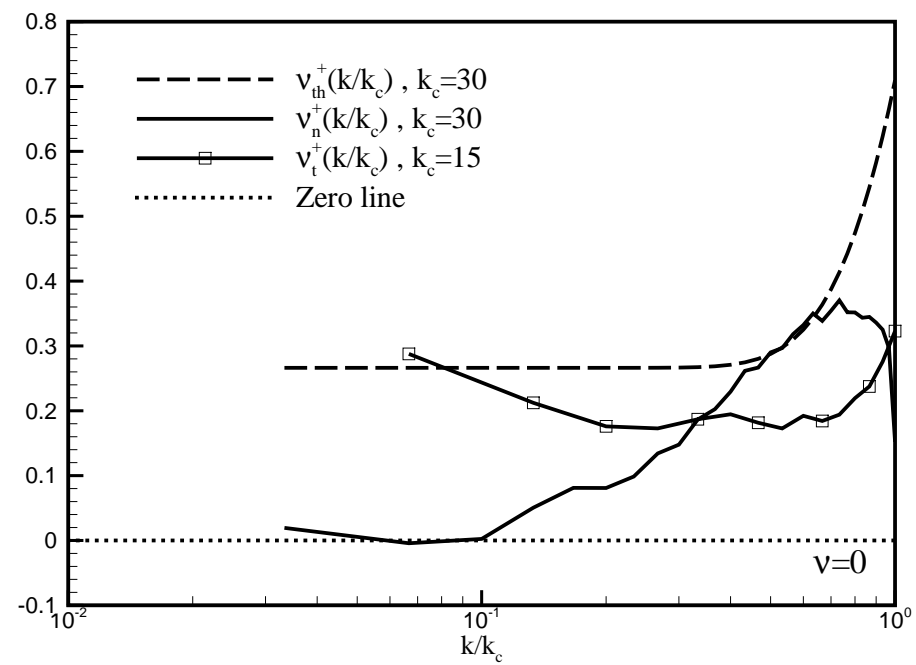

FIG. 1: Eddy viscosities for homogeneous isotropic turbulence. Broken line: theoretical eddy viscosity; solid line with symbols: intrinsic eddy viscosity for $k_{c}=15$; solid line: numerical eddy viscosity. The intrinsic and numerical eddy viscosities obtained from an inviscid simulation using MPDATA on a $64^{3}$ mesh. 